\title{
COMPARATIVE GENOME OF TWO STRAIN MORAXELLA CATARRHALIS USING IN SILICO ANALYSIS
}

\author{
Oktira Roka Aji \\ Department of Biology, Universitas Ahmad Dahlan \\ Jl. Prof. Dr. Soepomo, S.H. Yogyakarta 55164 \\ * Corresponding author: oktira.aji@bio.uad.ac.id
}

\begin{abstract}
Moraxella catarrhalis can cause otitis media and exacerbations of chronic obstructive pulmonary disease in human. Here we describe the comparison between two publicly available genomes of two strain of M.catarrhalis using computational analysis to obtain genomic features between them. Comparative genomic analysis were carried out using available tools in public domain websites. The aim of this study was to investigate the differences and similarities between two strains by comparing their genomic sequences. The results indicated that may be used to offer better understanding M.catarrhalis lifestyle.
\end{abstract}

Keywords: Moraxella catarrhalis; In Silico; Comparative genome analysis

\section{INTRODUCTION}

Comparative genome analysis is a branch of biology that studies the comparison between two different genomes. This approach can be used to study the bacterial lifestyle, virulences and host pathogen interaction. This analysis is based on the hypothesis that an important biological order has sustainable properties [1].

Moraxella catarrhalis is human-restricted respiratory tract pathogen. This pathogen cause otitis media and exacerbations of chronic obstructive pulmonary disease [2]. $\mathrm{M}$. catarrhalis strain BBH18 (formerly known as RH4 strain) is the first completely assembled genome sequence of this species [2]. The BBH18 was isolated from blood of an infected patient [2]. Wherease, M. catarrhalis strain CCRI-195ME was isolated from the middle ear of sick children with otitis media [3].

\section{MATERIAL AND METHOD}

Strain genome were downloaded from NCBI (National Center for Biotechnology Information) at www.ncbi.nlm.nih.gov/genome. Genome features of each strain was obtain from NCBI. BLAST Genome was used to analyze genome stability. The genomes sequences of the two strains were subjected to wgVISTA at genome.lbl.gov/vista/index.shtml [4] to identify conserved region between sequences. Phylogenetic tree was built using Mega7 [5] with bootstrap 1000 .

\section{RESULTS AND DISCUSSION \\ Genome features}

Genome features comparison can be seen in Table 1. Both genome features have no significant difference. However, M. catarrhalis strain CCRI-195ME has a plasmid. This plasmid contains 44 predicted open reading frames (ORF) [3]. 


\section{Whole genome alignment}

BLAST Genomes was used to analyze genome stability. Both strain share $99 \%$ identities (173843/174937) with 141/174937 gaps. Based on the genomic information, M. catarrhalis strain BBH18 has 343 predicted ORFs while M. catarrhalis strain CCRI-195ME has 377 predicted ORFs.

Conserved region was analysis using wgVista. Based on wgVISTA analysis, several regions in the genomes alignment were identified as divergent region (Figure 1). ORFs that located in those region were analyzed further to predict the protein content using Blastp (Table 2).

\section{Phylogenetic analysis}

In this phylogenetic analysis, we used five available M. catarhalis genome data in NCBI. Phylogenetic tree was built based on 16S rRNA gene sequences. 16S rRNA gene sequences were extracted from the genomes based on NCBI's annotation. Figure 2 show M. catarrhalis strain BBH18 was closer to 25239, FDAARGOS 213, and 25240 strain than CCRI-195ME.

\section{CONCLUSION}

In this report, M. catarrhalis strain BBH18 and CCRI-195ME genome were compared with each other. Both strain share several similarity and difference that makes each strain unique. We hope through this comparative genome analysis can contribute to gain deeper understanding of M. catarrhalis lifestyle.

\section{ACKNOWLEDGEMENTS}

We are grateful to Ministry of Research, Technology and Higher Education of the Republic of Indonesia for financial support for this project. This work was also supported by Universitas Ahmad Dahlan.

\section{REFERENCES}

[1] M. A. Nobrega and L. A. Pennacchio, 2003, J Physiol, vol. 554, no. 1, pp. 31-39.

[2] S. P. W. De Vries, S. A. F. T. Van Hijum, W. Schueler, K. Riesbeck, J. P. Hays, P. W. M. Hermans, and H. J. Bootsma, 2010, J. od Bacteriol., vol. 192, no. 14, pp. 35743583.

[3] A. Tan, L. V Blakeway, L. O. Bakaletz, M. Boitano, T. A. Clark, J. Korlach, M. P. Jennings, I. R. Peak, and L. Seib, 2017, Genome Announc., vol. 5, no. 21, pp. 16-17.

[4] Brudno M, Malde S , Poliakov A et al., 2003, Bioinformatics. 19:1 i54-i62

[5] Kumar, S. Stecher G. And Tamura K., 2016, Molecular Biology and Evolution 33: 1870-1874 
Table 1 Genome features of M. catarrhalis strain BBH18 and CCRI-195ME

\begin{tabular}{lcc}
\hline \multicolumn{1}{c}{ Features } & $\begin{array}{c}\text { Moraxella catarrhalis } \text { strain } \\
\text { BBH18 }\end{array}$ & $\begin{array}{c}\text { Moraxella catarrhalis } \text { strain } \\
\text { CCRI-195ME }\end{array}$ \\
\hline Genome size $(\mathrm{Mb})$ & 1.86329 & 1.99439 \\
GC content $\%$ & 41.70 & 41.56 \\
Genes & 1733 & 1900 \\
Protein & 1622 & 1775 \\
Ref sequences & NC_014147.1 & NZ.CP018059.1 \\
rRNA & 12 & 12 \\
tRNA & 50 & 50 \\
Other RNA & 4 & 4 \\
Pseudogene & 45 & 59 \\
Plasmid & 0 & 1 \\
Clinical details & Blood isolate & Middle ear \\
Source & Vries, 2010 & Tan, 2017 \\
\hline
\end{tabular}

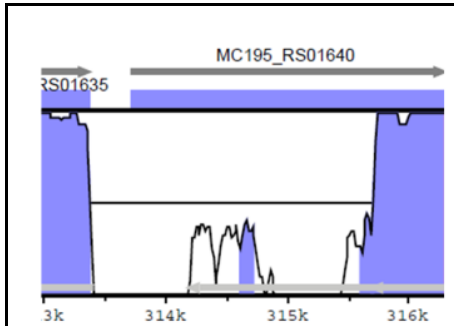

(A)

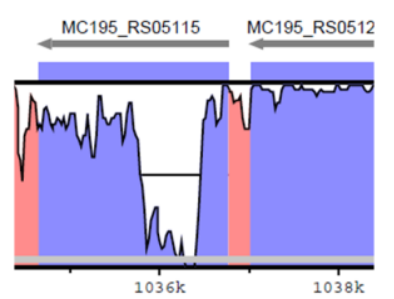

(D)

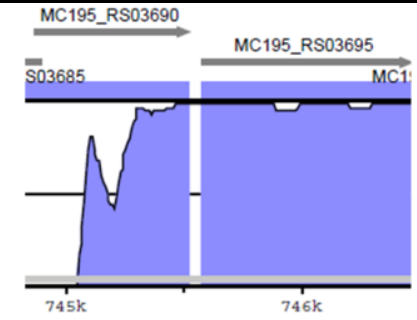

(B)

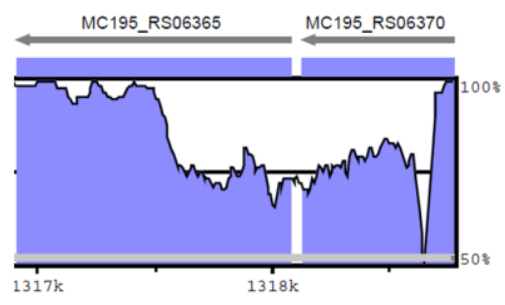

(E)

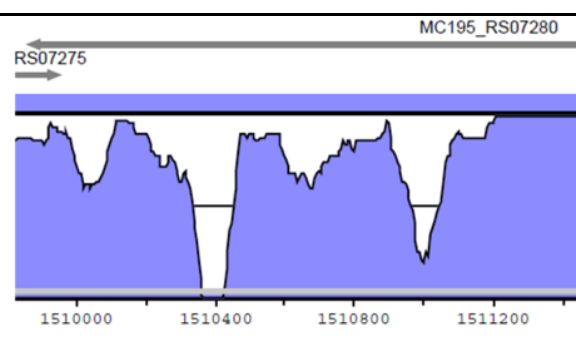

(C)

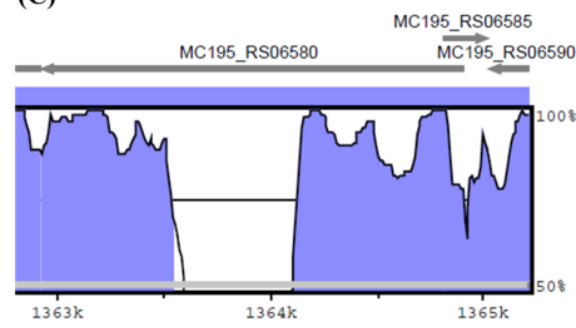

(F)

Figure 1 Graphical represent of M. catarrhalis strain BBH18 and CCRI-195ME genome analysis using wgVista. Blue colors represent coding sequences. Red colors represent noncoding sequences. 
Table 2 Protein encode by ORFs in the divergent region.

\begin{tabular}{ll}
\hline & ORF \\
\hline MC 195_RS01640 & ATP-dependent chaperone ClpB \\
MC195_RS03690 & peptidase S24 \\
MC 195_RS07280 & transferrin-binding protein \\
MC 195_RS05115 & transferrin-binding protein \\
MC 195_RS06365 & aspartate carbamoyltransferase \\
MC 195_RS06370 & sel1 repeat family protein \\
MC 195_RS06580 & site-specific DNA-methyltransferase \\
\hline
\end{tabular}

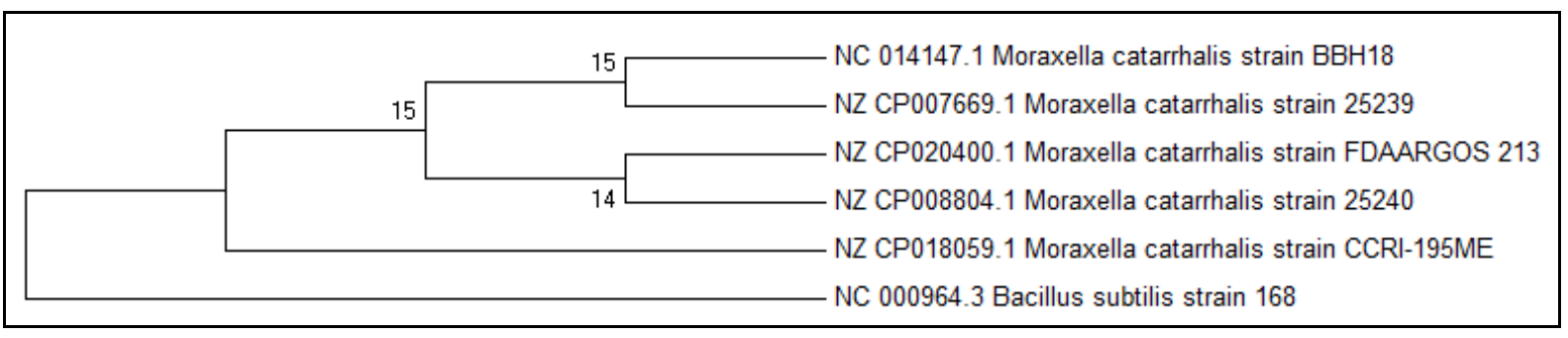

Figure 2 Phylogenetic tree of M. catarrhalis 16S rRNA gene sequences. 